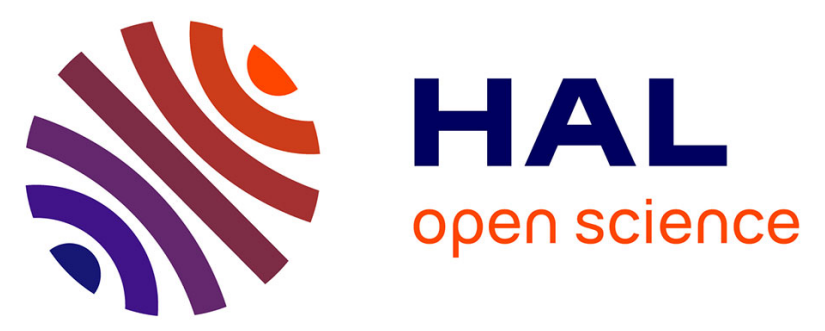

\title{
Near-infrared fluorescence imaging-guided surgery improves recurrence-free survival rate in novel orthotopic animal model of head and neck squamous cell carcinoma
}

Ihab Atallah, Clément Milet, Maxime Henry, Véronique Josserand, Emile P. Reyt, Jean-Luc Coll, Amandine Hurbin, Christian A. Righini

\section{To cite this version:}

Ihab Atallah, Clément Milet, Maxime Henry, Véronique Josserand, Emile P. Reyt, et al.. Near-infrared fluorescence imaging-guided surgery improves recurrence-free survival rate in novel orthotopic animal model of head and neck squamous cell carcinoma. Head \& Neck, 2016, 38 (S1), pp.E246-E255. 10.1002/hed.23980 . hal-02349412

\section{HAL Id: hal-02349412 \\ https://hal.science/hal-02349412}

Submitted on 8 Nov 2019

HAL is a multi-disciplinary open access archive for the deposit and dissemination of scientific research documents, whether they are published or not. The documents may come from teaching and research institutions in France or abroad, or from public or private research centers.
L'archive ouverte pluridisciplinaire HAL, est destinée au dépôt et à la diffusion de documents scientifiques de niveau recherche, publiés ou non, émanant des établissements d'enseignement et de recherche français ou étrangers, des laboratoires publics ou privés. 
Near-infrared fluorescence imaging-guided surgery improves the recurrence-free survival rate in a novel orthotopic animal model of HNSCC

Ihab Atallah MD-Msc ${ }^{1-3}$, Clément Milet ${ }^{1,2}$, Henry Maxime ${ }^{1,2}$, Véronique Josserand $\mathrm{PhD}^{1,2}$, Emile Reyt $\mathrm{MD}^{2,3}$, Jean-Luc Coll $\mathrm{PhD}^{1,2}$, Amandine Hurbin $\mathrm{PhD}^{1,2} *$, Christian Adrien Righini MD-PhD ${ }^{1-3} *$

*These authors contributed equally to this work.

\section{Affiliation of authors:}

1. INSERM U823, BP 170, 38042 Grenoble Cedex 9, France

2. Joseph Fourier University, BP 53, 38041 Grenoble Cedex 9, France

3. Department of Otolaryngology-Head and Neck Surgery, BP 217, Grenoble University Hospital, France Conflict of interest: none.

\section{For correspondence and request for reprints:}

Ihab Atallah

Department of Otolaryngology-Head and Neck Surgery

BP 217

Grenoble University Hospital

France

Tel.: +33 (0) 613743013

Fax: +33(0) 476549454

Mail: IAtallah@chu-grenoble.fr

Running title: HNSCC optical guided surgery in a novel orthotopic model

Keywords: Head and neck squamous cell carcinoma; orthotopic animal model, near-infrared optical imaging-guided surgery, $\alpha v \beta 3$ integrin; surgical margins 


\section{Abstract}

\section{Background}

Appropriate animal models are required to test novel therapeutics for head and neck squamous cell carcinoma (HNSCC) such as near-infrared (NIR) imaging-guided surgery.

\section{Methods}

We developed an optimized animal model of orthotopic HNSCC (in female athymic NMRI nude mice) with a prolonged survival time. Resection of the orthotopic tumors was performed 30 days after implantation with or without the aid of a miniaturized clinical grade NIR optical imaging device, after systemic administration of a fluorescent RGD-based probe that targets $\alpha_{\mathrm{v}} \beta_{3}$ integrin.

\section{Results}

NIR optical imaging-guided surgery increased the recurrence-free survival rate by $50 \%$ through the detection of fluorescent cancer residues as small as $185 \mu \mathrm{m}$; these fragments could remain unidentified if resection was performed exclusively under unaided visual guidance.

\section{Conclusion}

NIR optical imaging-guided surgery showed an improved HNSCC tumor resection quality in our optimized orthotopic animal model. 


\section{Introduction}

Head and neck squamous cell carcinoma (HNSCC) is the sixth most common cancer worldwide, with 600000 new cases diagnosed each year. Surgical resection is considered the cornerstone of HNSCC treatment and could be associated with radiochemotherapy in some cases. Although recent advances in the treatment of HNSCC have improved the survival rate, there is a considerable risk of mortality; with more than 300000 deaths from this disease annually (1-3).

The complete excision of HNSCC during surgical resection with negative surgical margins improves prognosis and decreases the recurrence rate (4). Intraoperative margin assessment of HNSCC is performed by visual inspection and palpation, followed by the selective assessment of any suspicious areas using rapid histological evaluation, which can be time consuming and sometimes inaccurate. Frequent discrepancies between the selective intraoperative assessment and the more comprehensive postoperative pathology margin assessment are not unusual, necessitating additional surgeries to achieve negative margins or postoperative radiochemotherapy, which can have multiple side effects.

One novel tool to improve tumor resection quality is near-infrared (NIR) optical imagingguided surgery, which creates a specific contrast between normal and cancer tissue, thus helping to achieve tumor free surgical margins. Multiple animal studies and clinical trials have been performed to determine the effectiveness of NIR optical guided surgery at optimizing the quality of brain, breast, skin and liver tumors resection, and these studies have reported many encouraging results (5-8). One of the most important targets of NIR optical imaging-guided surgery is $\alpha_{\mathrm{v}} \beta_{3}$ integrin, which plays an important role in cancer-associated 
angiogenesis, cell proliferation, migration and metastasis. It is widely expressed on neoendothelial cells and some cancer cells, such as HNSCC (9-13). RAFT-c(-RGDfK-) 4 (regioselectively addressable functionalized template-arginine-glycine-aspartic acid) is a peptide-like scaffold that contains four cyclo(-RGDfK-) (cRGD) motifs, and this molecule has been shown to target $\alpha_{\mathrm{v}} \beta_{3}$ integrin in vitro and in vivo. This peptide is also particularly efficient at targeted delivery of drugs and imaging contrast agents.(14-17)

The development and evaluation of novel anticancer agents or new surgical techniques for HNSCC treatment require the use of an appropriate animal model that must be representative and reproducible and can accurately recapitulate the disease process.

Here, we developed an experimental xenograft orthotopic animal model of HNSCC that can be used to test novel molecular therapeutics or surgical tools. In this model, orthotopic tumor implantation and resection was performed in a novel way. The aim of the development of our model is to assess the role of NIR optical imaging-guided surgery in the surgical resection of HNSCC tumors, by using a miniaturized NIR optical device designed for oral oncologic procedures (18) and fluorescent RAFT-c(-RGDfK-) 4 injection. Our orthoptopic model allowed us to test the benefits of NIR optical imaging-guided tumor resection compared to macroscopic tumor resection in the detection of cancer residues and its effect on the recurrence-free survival rate of mice.

\section{Materials and methods}

\section{Cell line:}


This study was conducted using the human head and neck squamous cell carcinoma cell line CAL33, which was stably transfected with the pLenti-luciferase vector. This cell line was obtained from the Antoine-Lacassagne Center, Nice, France (19). The cell line was maintained as a monolayer culture in DMEM supplemented with $10 \%$ FBS (v/v) in a humidified incubator (Sanyo, Japan) at $37{ }^{\circ} \mathrm{C}$ in an atmosphere containing $8 \% \mathrm{CO}_{2}$. CAL33 cells were used in vivo for establishing subcutaneous tumors from which tumor fragments were obtained for orthotropic tumor implantation. In addition to luciferase expression by these cells which allows bioluminescence imaging, they represent an adequate cellular model for HNSCC as they show positive expression of EGFR, IGF1R and NRP1 like in some types of head and neck cancers (20-24).

\section{Molecule}

AngioStamp ${ }^{\mathrm{TM}} 800$ (Fluoptics, Grenoble, France) was injected into the tail vein of the mice. This imaging agent is a tetravalent RGD-based peptide (RAFT-c(-RGDfK-) $)_{4}$ ) that targets $\alpha_{\mathrm{v}} \beta_{3}$ integrin. It is labeled with a NIR organic fluorophore with an absorbance and an emission maxima of $781 \mathrm{~nm}$ and $794 \mathrm{~nm}$ respectively to allow in vivo NIR fluorescence imaging using the NIR fluorescence imaging system described below $(15,17,25-27)$.

\section{Flow cytometry}

The expression of $\alpha_{v} \beta_{3}$ integrin and the binding of RAFT-c(-RGDfK-) $)_{4}$ to the CAL33 cell line were analyzed by flow cytometry. Briefly, CAL33 cells were harvested, washed with PBS containing $1 \mathrm{mM} \mathrm{CaCl}$ and $1 \mathrm{mM} \mathrm{MgCl}_{2}$ (PBS $\mathrm{Ca} 2+/ \mathrm{Mg} 2+$ ) and fixed in $2 \%$ paraformaldehyde for 15 minutes at room temperature. Cell fixation was done exclusively for the assessment of $\alpha_{v} \beta_{3}$ integrin expression. After washing twice with PBS Ca2+/Mg2+, the cells were incubated with an anti-human CD51/CD61 mouse phycoerythrin-conjugated 
antibody (clone 23C6, Pharmingen, BD Bioscience) or with the RAFT-c(-RGDfK-) 4 for 1 hour at $4{ }^{\circ} \mathrm{C}$ for the former and at $37{ }^{\circ} \mathrm{C}$ for the later, then washed again and resuspended in PBS Ca2+/Mg2+ at $4{ }^{\circ} \mathrm{C}$. Analysis was performed using a BD Accuri C6 flow cytometer (BD Biosciences, US) and the CFlow Plus software. The results are reported as the mean fluorescence intensity. The RGD-based peptide RAFT-c(-RGDfK-) $)_{4}$ used in the flow cytometry analysis was labeled by $\mathrm{Cy} 5$ to make it compatible with the laser wavelength and filters of BD Accuri C6 flow cytometer. Cy5-labeled RAFT-c(-RGDfK-) 4 was synthesized according to previously reported procedures (28).

\section{Animals}

All animal experiments were performed in agreement with the EEC guidelines and the Principles of Laboratory Animal Care (NIH publication 14, nos. 86-23, revised 1985); the protocol was approved by the Animal Care and Use Committee (Comité d'éthique en expérimentation animale de Grenoble). Female athymic NMRI nude mice (Janvier, Le Genest-Isle, France) were maintained under specific pathogen-free conditions. The animals were housed and optical imaging was performed at the small animal optical imaging platform OPTIMAL of INSERM U823 Institute.

\section{Subcutaneous tumor implantation and excision}

Human CAL33 cells were harvested, and $10^{6}$ cells in PBS were injected subcutaneously into the flank of the mice for heterotropic establishment of tumors. Tumor growth was quantified by measuring the tumors in two dimensions with a Vernier calliper twice a week. The tumor volume was calculated using the following equation: $\mathrm{a} \times \mathrm{b}^{2} \times 0.4$, where $\mathrm{a}$ and $\mathrm{b}$ are the largest and smallest diameters, respectively. When the tumor volumes reached $750 \mathrm{~mm}^{3}$, after approximately 14 to 16 days, the tumors were excised by direct approach under general 
anesthesia with isoflurane/oxygen; $3.5 \%$ anesthesia was used for induction, and $1.5 \%$ was used for the maintenance of anesthesia. The mice were then euthanized by cervical dislocation. Fragments from these tumors were subsequently used for orthotopic tumor implantation as described below.

\section{Orthotopic tumor implantation}

Under general anesthesia, obtained by intraperitoneal injection of Domitor (Pfizer, Orsay, France) and Imalgene (Merial, Lyon, France), a small incision was performed on the inner aspect of the mouse's cheek. A tumor fragment with a diameter of $0.5 \mathrm{~mm}$, obtained from the subcutaneous tumours, was implanted through the incision, and the incision was closed using

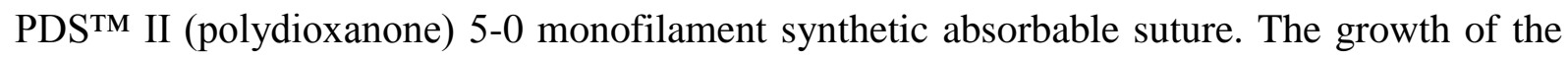
orthotopic tumors was examined each week using non-invasive bioluminescence imaging, and the tumors reached the maximal size compatible with the mouse's well-being 30 days after implantation.

\section{Bioluminescence imaging}

The mice received an intraperitoneal injection of luciferin $(150 \mathrm{mg} / \mathrm{kg}) 5 \mathrm{~min}$ before the bioluminescence imaging, which was carried out using an IVIS Kinetic device (Caliper Life Sciences). This enabled the quantification and tracing of the bioluminescent signal during orthotopic tumor development and the early detection of recurrence after tumor resection. Semiquantitative data were obtained from the bioluminescence images within demarcated regions of interest in the area to be quantified. The results were expressed as the number of photons per second.

\section{NIR fluorescence imaging}


AngioStamp $^{\mathrm{TM}} 800$ was injected into the tail vein of the mice at a dose of $10 \mathrm{nmol}$ per mouse 16 to 24 hours before fluorescence imaging to obtain the maximal fluorescence signal

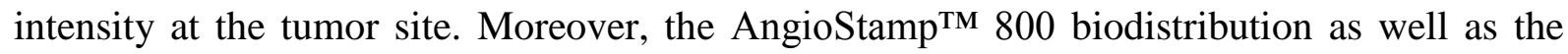
orthotopic tumors fluorescence kinetics was performed. NIR fluorescence imaging was performed using the FluoStick ${ }^{\mathrm{TM}}$ Clinical System (Fluoptics, Grenoble, France). This novel commercial fluorescence imaging system has been miniaturized and optimized for oral oncologic procedures in humans. It excites fluorescence at $750 \mathrm{~nm}$ and detects the emitted light at wavelengths greater than $800 \mathrm{~nm}$.

\section{Orthotopic tumor resection}

Orthotopic tumor resection was performed under visual guidance or through the aid of NIR optical imaging. Under general anesthesia (intraperitoneal injection of Domitor and Imalgene), we performed a direct approach through an incision on the outer aspect of the cheek to obtain a large surgical field that permitted tumor dissection and resection. Initially, the tumor was exposed, and the fluorescence of the head and neck region was obtained. Intentionally, 90\% of the tumor was removed under visual guidance, without NIR optical imaging, and a rim of tissue $(10 \%)$ was left behind to simulate residual disease and to investigate the ability of the fluorescence imaging to detect smaller disease foci. The rim of the residual tumor tissue was then removed under visual guidance to achieve a complete macroscopic tumor resection. After excision, fluorescence imaging was repeated. In this step, any residual fluorescent tissue unintentionally left behind was removed with the help of the fluorescence imaging guidance system. Non-fluorescent control tissue fragments were also taken from the tumor bed. One dose of $0.1 \mathrm{mg} / \mathrm{kg}$ buprenorphine (RB Pharmaceuticals, France) was injected subcutaneously to reduce postoperative pain after orthotopic tumor resection. The fluorescence of the tumor, residual fluorescent tissue and control tissue were measured ex vivo. 


\section{Analysis of NIR fluorescence data}

The fluorescent data collected using the FluoStick ${ }^{\mathrm{TM}}$ Clinical System were quantified with the WASABI Imaging software V.1.5.0.5 (Hamamatsu Photonics GmbH, Germany) and Microsoft Excel. The fluorescence intensity value of the different tissues was defined as the mean fluorescence intensity of the region of interest minus the mean fluorescence intensity of the background divided by the exposure time in milliseconds, resulting in the relative light unit per pixel per millisecond (RLU/pixel/ms).

\section{Mouse follow-up after tumor resection}

Four mice were used experimentally to develop the orthotopic model. The rest of mice $(n=24)$ were examined and weighed regularly after orthotopic tumor excision to detect tumor recurrence through repeated clinical examination and bioluminescence imaging in order to compare the recurrence-free rate of the macroscopic tumor resection and the NIR fluorescence imaging-guided tumor resection. Any mouse that showed signs of tumor recurrence was euthanized by cervical dislocation. Mice without relapse were followed up for two months after tumor excision and were then euthanized and dissected post-mortem to search for infra-clinical recurrence.

\section{Histopathological and immunohistochemical analyses}

Surgical specimens were separated into two samples for histopathological and immunohistological analyses of the $\alpha_{v} \beta_{3}$ integrin expression level. A portion of the samples was fixed in formaldehyde, embedded in paraffin and cut into $7 \mu \mathrm{m}$ slices for hematoxylin and eosin (H.E.) staining of the orthotopic tumors, residual fluorescent tissues and non-fluorescent control tissues. Histological examination was performed under an Olympus BX41 Laboratory 
Microscope. Any tumor foci identified in the residual fluorescent tissues were measured using the Image $\mathrm{J}$ 1.46r software. Other samples were frozen in isopentane and underwent shock freezing in liquid nitrogen. These samples were cut into $8 \mu \mathrm{m}$ slices (microcryotome) and stored at $-80{ }^{\circ} \mathrm{C}$ until fixation and staining. After the frozen slices were thawed at room temperature, they were fixed in acetone. Immunostaining with a mouse anti-human integrin $\alpha_{v} \beta_{3}$ monoclonal antibody (clone MAB1976, 1:500; Millipore) was performed on the acetonefixed cryosections of the orthotopic tumours using the M.O.M. immunodetection (peroxidase) Kit (Vector laboratories, Inc., Burlingame, CA). Staining was performed using liquid DAB and a substrate chromogen system (Dako, North America, Inc., CA). The nuclei were counterstained with hematoxylin.

\section{Statistical analysis}

Statistical analysis was performed using the coefficient of determination $R^{2}$ in the GraphPad Prism 5 software (GraphPad Software, Inc.). Statistical significance was assigned for values of $p<0.05$.

\section{Results}

\section{Development of an optimized head and neck cancer orthotopic animal model}

Twenty eight mice were implanted with a tumor fragment obtained from subcutaneous CAL33 tumors (fig. 1a and 1b). All mice survived after the orthotopic tumor implantation, and there were no complications during the surgical procedure. Minimal cheek swelling and inflammation was observed after implantation, and this regressed spontaneously after one day. The mice did not exhibit weight loss, and there was no change in feeding during tumor development (fig. 1f). Orthotopic tumors could be observed macroscopically from day 10 to 
12 following implantation (fig. 1c). The mice underwent orthotopic tumor resection on the day 30 day after implantation (fig. 1d). There were no complications during tumor excision except that special care had to be taken to ensure hemostasis, by applying pressure with a cotton swab to stop the bleeding and to prevent blood inhalation by the mice during surgery. One mouse developed a cheek hematoma that spontaneously resolved postoperatively. Minimal transient weight loss of about two grams was observed in some mice following tumor resection owing to difficulty in feeding due to the presence of cheek inflammation; this condition spontaneously resolved after 3 days (fig. 1f), and weight gain returned to its normal rate within 2 days of explantation. All mice survived after tumor resection, and complete wound healing was finished after 5-7 days (fig. 1e). Pathological analysis of resected orthotopic tumors showed the characteristics of moderately differentiated HNSCC (fig. 2a).

\section{Expression of $\alpha_{v} \beta_{3}$ integrin in the CAL33 cell line}

Flow cytometry analysis showed a positive in vitro expression of $\alpha_{v} \beta_{3}$ integrin by $98.5 \%$ of the CAL33 cells (fig. 2b). RAFT-c(-RGDfK-) 4 showed a positive binding to $96.2 \%$ of these cells (fig. 2c). In vivo expression of $\alpha_{v} \beta_{3}$ integrin in the human CAL33 cell line was also confirmed by immunohistochemical labelling of the tumor sections; positive staining in tumor samples (fig. 2d) and negative staining in control tissue (fig. 2e).

These results were essential to target the orthotopic tumors developed from this cell line using the RGD-based probe AngioStamp ${ }^{\mathrm{TM}} 800$ NIR Molecular Imaging Agent, which binds specifically to $\alpha_{\mathrm{v}} \beta_{3}$ integrin.

\section{Fluorescence imaging is a valuable tool for head and neck tumor resection}


The biodistribution of AngioStamp ${ }^{\mathrm{TM}} 800$ showed high concentration in the kidney and in the orthotopic tumor 24 hours after intravenous injection (fig 3a). Importantly, elevated tumor tumor to background fluorescence ratios were obtained 16 to 24 hours after AngioStamp ${ }^{\mathrm{TM}}$ 800 injection (fig. 3b). Two independent group of mice ( $n=6$ in each group) underwent tumor resection with the aid of fluorescence imaging as described in the materials and methods section. All orthotopic tumors were fluorescent (fig. 4a) with a very elevated contrast of 65 ( \pm 22) due to the high fluorescence ratio of the tumor to the surrounding muscle. After macroscopic excision of $90 \%$ of the tumor, the FluoStick ${ }^{\mathrm{TM}}$ Clinical System device (fig. $4 \mathrm{f}$ ) detected the residual $10 \%$ of the tumor (fig. $4 \mathrm{~b}$ ). This confirmed that fluorescence imaging could efficiently detect small disease foci. After removal of the $10 \%$ macroscopic tumor residue under visual guidance, residual fluorescent tissue $0.2-1.8 \mathrm{~mm}$ in diameter was unintentionally left behind in all of the mice (fig. $4 c)$. These tissue foci $(n=37)$ were removed under fluorescence imaging guidance. No fluorescent signal could be detected in the tumor bed after the residual fluorescent tissue excision (fig. 4d), except for the autofluorescence signal of the mandible and of the skin that was observed in a few mice. All resected tissue foci were significantly fluorescent ex vivo compared to the control tissue (fig. 4e). Histopathological analysis (fig. 5) showed that tumor foci were present in $94.6 \%$ of the residual fluorescent tissues. For each mouse, at least one fluorescent residue revealed a tumor focus. Histological examination showed that fluorescence imaging could detect tumor foci as small as $185 \mu \mathrm{m}$ (fig. 5a). There was a positive correlation between the diameter of the tumor focus and the fluorescence intensity of the residual tissue containing the focus (fig. 5b). Moreover, the fluorescent probe provided good contrast between residual cancer tissue and normal tissue with a residual cancer tissue to background tissue fluorescence ratio of $10( \pm 6)$.

\section{Effect of NIR optical imaging-guided surgery on recurrence-free survival rate}


The recurrence-free survival rate of mice after NIR optical imaging-guided surgery was compared to that after visually guided macroscopic tumor resection. Bioluminescence was performed regularly after tumor resection (fig. 6a). In two independent groups where the resection of orthotopic tumors was performed under visual guidance ( $n=6$ in each group), the macroscopic recurrence of the disease was mostly observed 3-4 weeks after tumor excision in $75 \%( \pm 5)$ of the mice, in contrast to $25 \%( \pm 5)$ in the two groups where NIR optical imaging was used during tumor resection ( $\mathrm{n}=6$ in each group) (fig. $6 \mathrm{~b}-\mathrm{d}$ ). Relapse was confirmed by bioluminescence, which showed an early gradual increase of the bioluminescent signal. No tumor was found on the post-mortem dissection of mice that showed no signs of macroscopic recurrence. Therefore, we found that NIR optical imaging-guided surgery increased the recurrence-free survival rate by $50 \%$ two months after tumor resection (fig. 6e).

\section{Discussion}

The advantage of orthotopic HNSCC animal models over subcutaneous models is that they provide specific interactions between the cancer cells and their native environment. These interactions can influence the molecular, pathological, and clinical features of the orthotopic tumors, rendering them representative of human HNSCC tumours (29-31).

Mice are one of the preferred animal species in biomedical research because they are small in size, easy to handle and relatively inexpensive. Several methods have been used to develop orthotopic HNSCC mouse models. One of these methods is based on the repetitive local application of carcinogenic agents, such as 7,12-dimethylbenz[a]anthracene (DMBA), 4nitroquinoline 1-oxide (4NQO), 12-O-tetradecanoylphorbol-13-acetate, benzo[a]pyrene, 4(methylnitrosamino)-1-(3-pyridyl)-1-butanone and nitrosonornicotine, in the oral cavity. The 
major disadvantage of this method is that it takes between 12 and 34 weeks to develop tumours with a poor penetrance. Additionally, these tumors do not mimic the invasive pathology of HNSCC in humans (29-32). In a transgenic mouse model of HNSCC, the keratin 5 (K5) or keratin 14 (K14) promoters are used to overexpress the oncogene K-ras ${ }^{\mathrm{G} 12 \mathrm{D}}$ in the oral epithelium to induce squamous cell carcinoma (33-34). Although these transgenic models appear to reproduce some clinical characteristics of HNSCC, one major point that should be taken into consideration is that no single gene is predominantly associated with the HNSCC carcinogenic process in humans. In addition, the frequency of K-ras mutations in HNSCC is relatively low (31). Finally, one of the most important methods of inducing head and neck tumors in animals is through the orthotopic xenograft of HNSCC by injecting the cancer cells into the tongue or mouth floor of the mouse. The major disadvantage of this model is that animals have to be euthanized on day 10 to 12 after tumor cell injection because of rapid tumor growth that prevents correct feeding by the animals, resulting in unethical suffering (21, 35-36). This short period with in situ tumors does not allow for the adequate long-term efficacy evaluation of new treatments. Additionally, due to the disruption of vital structures in the oral cavity during tumor resection, the mice have to be euthanized at the end of the surgery, which prevents the evaluation of the post-operative benefits and effects of novel surgical and therapeutic tools.

We developed a novel method of orthotopic HNSCC tumor implantation and resection in female athymic NMRI nude mice. Our model allowed us to obtain a period of 30 days with in situ tumors. Our method used tumor fragment implantation instead of direct cellular injection and was developed in other types of small animal xenograft tumor models for gastric, hepatic and oesophageal cancers (37-39). This method prevented the cancer cell dissemination created by the hydrostatic pressure of the syringe during injection, which results in the rapid 
development of large tumors that reduce mice survival to 10-12 days (29-31). All mice that underwent tumor fragment implantation developed an orthotopic tumor. A major advantage of our model is that orthotopic tumor resection can be performed 30 days after implantation. Furthermore, the postoperative survival rate reached $100 \%$ because the site of orthotopic tumor development and our surgical resection technique preserved the vital structures of the mouse oral cavity. This allowed us to follow up with the mice when investigating novel surgical or medical treatments to establish efficacy, toxicity, and survival studies.

The prognosis of HNSCC depends on many factors, such as the TNM staging, the localization of the tumor, the level of differentiation and the quality of the surgical margins. In HNSCC, as in other solid tumors, complete tumor resection improves the patient's outcome. Positive surgical margins cannot be compensated by adjuvant radiotherapy/chemotherapy, and they increase the rate of tumor recurrence and reduce the overall survival rate (4). In real life surgical practice, to ensure tumor removal, the surgeon excises a safe margin of 0.5 to $1.0 \mathrm{~cm}$ around the tumor. This margin is difficult to determine macroscopically at the deepest part of the tumor, which is in contact with the surgical bed, because resection at this site depends mainly on palpation and not on visual guidance. The pathologist then uses frozen sections to confirm that the margins of resection do not contain cancerous tissue. Unfortunately, frozen sections removed at the level of the surgical bed lead to important sampling errors because of the increased difficulty in margin relocation. Consequently, small residual cancer foci can be left behind, resulting in an increased risk of tumor recurrence (40-41).

The introduction of real-time imaging technologies, such as NIR fluorescence, into the operating room has the potential of bridging the gap between radiology and surgery, resulting in intraoperative image-guided surgery that improves the quality of solid tumor resection. 
This type of imaging requires specific targeted contrast agents that bind to the cancer cells and generate a significant signal to noise ratio between the tumor and the surrounding normal tissue. Various cell surface molecules, including epidermal growth factor receptor, Her-2/Neu, $\alpha_{v} \beta_{3}$ integrin, urokinase plasminogen activator receptor, mucin 1, carcinoembryonic antigen, and the folate receptor, have been investigated for targeted imaging (8). Multiple studies have been performed in animals and humans to evaluate the role of NIR optical imaging-guided surgery in solid tumor resection. In preclinical animal models, NIR optical imaging-guided surgery was used to optimize the resection quality of breast, ovarian, brain and colon tumors or metastases. In these models, $\alpha_{\mathrm{v}} \beta_{3}$ integrin, which is overexpressed in tumor cells and in neo-endothelial cells, was the most common receptor targeted in NIR optical imaging-guided surgery. AngioStamp ${ }^{\mathrm{TM}}$ 800, the commercial version of the RAFT-c(-RGDfK-) ${ }_{4}$ peptide coupled with a fluorophore, was commonly used to target $\alpha_{v} \beta_{3}$ integrin. NIR optical imagingguided surgery in these models improved the tumor resection quality and the detection and excision of metastases $(5,42-44)$. Other studies have also been performed in humans to assess the benefit of NIR optical imaging-guided surgery in head and neck cancer resection. In most of these studies, ICG (indocynaine green) was used as a fluorescent tracer. Although ICG is considered a blood pool agent that is not inherently specific for any tumor, it is the only NIR fluorescent agent that has been used in clinical applications because it is EMA (European Medicines Agency) and FDA (US Food and Drug Administration) approved. Yoakoyama et al. used ICG to delineate head and neck tumors and lymph node metastases in humans 30 to 60 minutes after intravenous administration (45). All tumors and lymphatic metastases displayed bright fluorescence emissions that clearly contrasted with the normal structures. Fluorescence imaging was very useful to detect metastatic lymph nodes that were not palpable, and not visible macroscopically, in the retropharyngeal space. Despite of these encouraging results, precautions have to be taken in tumor margin demarcation using ICG because 
accumulation of ICG may not be limited to cancerous tissues. In fact, ICG would also be expected to accumulate in inflammatory tissues and areas of surgical trauma that could partly hamper specificity in the initial phase of enhanced permeability and retention effect. However, these effects occur at much lower levels and retention in tumors is greatly increased owing to defective architecture of lymphatic drainage (46-47). For these reasons, a targeted probe, like the one that we used in our study, would provide real-time, intra-operative distinction of the molecular edge between cancer and adjacent normal tissue. The benefit would be to potentially decreasing the incidence of a positive surgical tumor margin.

AngioStamp ${ }^{\mathrm{TM}} 800$ that targets specifically $\alpha_{\mathrm{v}} \beta_{3}$ integrin was used in our orthotropic model because $\alpha_{v} \beta_{3}$ integrin is expressed in HNSCC tumors (9-13), as well as in the CAL33 HNSCC cell line. We previously developed a miniaturized NIR optical imaging prototype adapted to the geometry of the oral cavity (18). In the present work, we used a commercial clinical grade device called FluoStick ${ }^{\mathrm{TM}}$, which was also developed for use in oral oncologic procedures in humans. After macroscopic removal of the orthotopic tumors, very small residual fluorescent tissues of $0.2-1.8 \mathrm{~mm}$ in diameter were unintentionally left behind, and these foci were detected in the tumor bed. These fluorescent residues were removed separately, and cancer foci as small as $185 \mu \mathrm{m}$ were identified in these extracted samples; a positive correlation between the intensity of the fluorescence and the size of the cancer focus existed. Indeed, the amount of bound AngioStamp ${ }^{\mathrm{TM}} 800$ increases as the number of malignant and neoendothelial cells in the tumor foci increases. Additionally, we showed that NIR optical imaging-guided surgery improved the recurrence-free survival rate by $50 \%$ two months after tumor resection. Because NIR optical imaging-guided surgery improved the quality of HNSCC resection and decreased the cancer recurrence rate in our orthotopic animal model, this valuable tool could be used in humans to optimize HNSCC resection, especially at the 
deepest part of the tumor, through the identification of small tumor foci, as shown in our model.

\section{Conclusion}

We developed a reproducible orthotopic mouse model of HNSCC with mice surviving up to 30 days with an in situ tumor, as well as after tumor resection. Our orthotopic model currently provides the most accurate reflection of the clinical situation and allows efficacy, toxicity, and survival studies of novel medical or surgical treatments for HNSCC. This model allowed us to demonstrate that NIR optical imaging-guided surgery improves the quality of HNSCC resection by detecting fluorescent cancer foci that could remain unidentified if resection was performed exclusively under visual guidance. This preclinical stage is the first step toward testing NIR optical imaging-guided surgery for HNSCC in humans.

\section{Acknowledgments}

This work was supported by the Fondation d'Entreprise Groupe Pasteur Mutualité, the Fondation Avenir and the Société Française d'ORL. We would also like to thank Dr. BOZEC (Antoine-Lacassagne Center, Nice, France) who provided the CAL33 cell line. We are very grateful to all of the team at the OPTIMAL-Grenoble small animal optical imaging platform of INSERM U823, particularly Dr. J Vollaire. Finally, we would like to thank Fluoptics for their technical assistance.

\section{References}


1. Kamangar F, Dores GM, Anderson WF. Patterns of cancer incidence, mortality, and prevalence across five continents: defining priorities to reduce cancer disparities in different geographic regions of the world. J Clin Oncol 2006;24(14):2137-50.

2. Hu D, Lau OD, Wang L, et al. A novel modular polymer platform for the treatment of head and neck squamous cell carcinoma in an animal model. Arch Otolaryngol Head Neck Surg 2012;138(4):412-7.

3. Mehanna H, Paleri V, West CM, Nutting C. Head and neck cancer-part 1: epidemiology, presentation, and preservation. Clin Otolaryngol 2011;36(1):65-8.

4. Eldeeb H, Macmillan C, Elwell C, Hammod A. The effect of the surgical margins on the outcome of patients with head and neck squamous cell carcinoma: single institution experience. Cancer Biol Med 2012;9(1):29-33.

5. Themelis G, Harlaar NJ, Kelder W, et al. Enhancing surgical vision by using real-time imaging of alphavbeta3-integrin targeted near-infrared fluorescent agent. Ann Surg Oncol 2011;18(12):3506-13.

6. Sexton K, Tichauer K, Samkoe KS, Gunn J, Hoopes PJ, Pogue BW. Fluorescent affibody Peptide penetration in glioma margin is superior to full antibody. PLoS One 2013;8(4):e60390.

7. Liu Y, Akers WJ, Bauer AQ, et al. Intraoperative detection of liver tumors aided by a fluorescence goggle system and multimodal imaging. Analyst 2013;138(8):2254-7.

8. Hadjipanayis CG, Jiang H, Roberts DW, Yang L. Current and future clinical applications for optical imaging of cancer: from intraoperative surgical guidance to cancer screening. Semin Oncol 2011;38(1):109-18.

9. Lu JG, Sun YN, Wang C, Jin de J, Liu M. Role of the alpha v-integrin subunit in cell proliferation, apoptosis and tumor metastasis of laryngeal and hypopharyngeal squamous cell 
carcinomas: a clinical and in vitro investigation. Eur Arch Otorhinolaryngol 2009;266(1):8996.

10. Danhier F, Le Breton A, Preat V. RGD-based strategies to target alpha(v) beta(3) integrin in cancer therapy and diagnosis. Mol Pharm 2012;9(11):2961-73.

11. Keereweer S, Mol IM, Kerrebijn JD, et al. Targeting integrins and enhanced permeability and retention (EPR) effect for optical imaging of oral cancer. J Surg Oncol 2012;105(7):714-8.

12. Fabricius EM, Wildner GP, Kruse-Boitschenko U, Hoffmeister B, Goodman SL, Raguse JD. Immunohistochemical analysis of integrins alphavbeta3, alphavbeta5 and alpha5beta1, and their ligands, fibrinogen, fibronectin, osteopontin and vitronectin, in frozen sections of human oral head and neck squamous cell carcinomas. Exp Ther Med 2011;2(1):919.

13. Takayama S, Hatori M, Kurihara Y, Kinugasa Y, Shirota T, Shintani S. Inhibition of TGF-beta1 suppresses motility and invasiveness of oral squamous cell carcinoma cell lines via modulation of integrins and down-regulation of matrix-metalloproteinases. Oncol Rep 2009;21(1):205-10.

14. Jin $\mathrm{ZH}$, Josserand $\mathrm{V}$, Razkin $\mathrm{J}$, et al. Noninvasive optical imaging of ovarian metastases using Cy5-labeled RAFT-c(-RGDfK-)4. Mol Imaging 2006;5(3):188-97.

15. Jin ZH, Josserand V, Foillard S, et al. In vivo optical imaging of integrin alphaV-beta3 in mice using multivalent or monovalent cRGD targeting vectors. Mol Cancer 2007;6:41.

16. Sancey L, Ardisson V, Riou LM, et al. In vivo imaging of tumour angiogenesis in mice with the alpha(v)beta (3) integrin-targeted tracer 99mTc-RAFT-RGD. Eur J Nucl Med Mol Imaging 2007;34(12):2037-47. 
17. Garanger E, Boturyn D, Jin Z, Dumy P, Favrot MC, Coll JL. New multifunctional molecular conjugate vector for targeting, imaging, and therapy of tumors. Mol Ther 2005;12(6):1168-75.

18. Gioux S, Coutard JG, Berger M, et al. FluoSTIC: miniaturized fluorescence imageguided surgery system. J Biomed Opt 2012;17(10):106014.

19. Bozec A, Sudaka A, Etienne-Grimaldi MC, Brunstein MC, Fischel JL, Milano G. Antitumor activity of cetuximab associated with the taxotere-cisplatin-fluorouracil (TPF) combination on an orthotopic head and neck cancer model. Oral Oncol 2011;47(10):940-5.

20. Barnes CJ, Ohshiro K, Rayala SK, El-Naggar AK, Kumar R. Insulin-like growth factor receptor as a therapeutic target in head and neck cancer. Clin Cancer Res 2007;13(14):4291-9.

21. Bozec A, Formento P, Lassalle S, Lippens C, Hofman P, Milano G. Dual inhibition of EGFR and VEGFR pathways in combination with irradiation: antitumour supra-additive effects on human head and neck cancer xenografts. Br J Cancer 2007;97(1):65-72.

22. Alattar M, Omo A, Elsharawy M, Li J. Neuropilin-1 expression in squamous cell carcinoma of the oesophagus. Eur J Cardiothorac Surg 2014;45(3):514-20.

23. Wang ZL, Tang ZC, Zhang Y, et al. Neuropilin-1 down-regulation impairs cell migration and induces the differentiation of human tongue squamous cell carcinoma cells. Head Neck Oncol 2012;4:54.

24. Liu X, Jiang L. [Present research on the status and the prospect of insulin-like growth factor-I in head and neck cancer]. Lin Chung Er Bi Yan Hou Tou Jing Wai Ke Za Zhi 2012;26(15):717-20.

25. Keramidas M, Josserand V, Feige JJ, Coll JL. Noninvasive and quantitative assessment of in vivo angiogenesis using RGD-based fluorescence imaging of subcutaneous sponges. Mol Imaging Biol 2013;15(3):239-44. 
26. Wenk $\mathrm{CH}$, Ponce F, Guillermet S, et al. Near-infrared optical guided surgery of highly infiltrative fibrosarcomas in cats using an anti-alphavss 3 integrin molecular probe. Cancer Lett 2013;334(2):188-95.

27. Boturyn D, Coll JL, Garanger E, Favrot MC, Dumy P. Template assembled cyclopeptides as multimeric system for integrin targeting and endocytosis. J Am Chem Soc 2004;126(18):5730-9.

28. Sancey L, Garanger E, Foillard S, et al. Clustering and internalization of integrin alphavbeta3 with a tetrameric RGD-synthetic peptide. Mol Ther 2009;17(5):837-43.

29. Cabanillas R, Secades P, Rodrigo JP, Astudillo A, Suarez C, Chiara MD. [Orthotopic murine model of head and neck squamous cell carcinoma]. Acta Otorrinolaringol Esp 2005;56(3):89-95.

30. Lu SL, Herrington H, Wang XJ. Mouse models for human head and neck squamous cell carcinomas. Head Neck 2006;28(10):945-54.

31. Kim S. Animal models of cancer in the head and neck region. Clin Exp Otorhinolaryngol 2009;2(2):55-60.

32. Aubry K, Paraf F, Monteil J, Bessede JP, Rigaud M. Characterization of a new rat model of head and neck squamous cell carcinoma. In Vivo 2008;22(4):403-8.

33. Caulin C, Nguyen T, Longley MA, Zhou Z, Wang XJ, Roop DR. Inducible activation of oncogenic K-ras results in tumor formation in the oral cavity. Cancer Res 2004;64(15):5054-8.

34. Vitale-Cross L, Amornphimoltham P, Fisher G, Molinolo AA, Gutkind JS. Conditional expression of K-ras in an epithelial compartment that includes the stem cells is sufficient to promote squamous cell carcinogenesis. Cancer Res 2004;64(24):8804-7. 
35. Bozec A, Sudaka A, Toussan N, Fischel JL, Etienne-Grimaldi MC, Milano G. Combination of sunitinib, cetuximab and irradiation in an orthotopic head and neck cancer model. Ann Oncol 2009;20(10):1703-7.

36. Bozec A, Sudaka A, Fischel JL, Brunstein MC, Etienne-Grimaldi MC, Milano G. Combined effects of bevacizumab with erlotinib and irradiation: a preclinical study on a head and neck cancer orthotopic model. Br J Cancer 2008;99(1):93-9.

37. Li Y, Li B, Xiang CP, Zhang Y, Li YY, Wu XL. Characterization of gastric cancer models from different cell lines orthotopically constructed using improved implantation techniques. World J Gastroenterol 2012;18(2):136-43.

38. Huang J, Zhang $\mathrm{Y}$, Zhong $\mathrm{H}$, et al. Comparison of endoscopic submucosal implantation vs. surgical intramuscular implantation of VX2 fragments for establishing a rabbit esophageal tumor model for mimicking human esophageal squamous carcinoma. PLoS One 2014;9(1):e85326.

39. Chen JH, Lin YC, Huang YS, Chen TJ, Lin WY, Han KW. Induction of VX2 carcinoma in rabbit liver: comparison of two inoculation methods. Lab Anim 2004;38(1):7984.

40. Meier JD, Oliver DA, Varvares MA. Surgical margin determination in head and neck oncology: current clinical practice. The results of an International American Head and Neck Society Member Survey. Head Neck 2005;27(11):952-8.

41. Ord RA, Aisner S. Accuracy of frozen sections in assessing margins in oral cancer resection. J Oral Maxillofac Surg 1997;55(7):663-9; discussion 669-71.

42. Harlaar NJ, Kelder W, Sarantopoulos A, et al. Real-time near infrared fluorescence (NIRF) intra-operative imaging in ovarian cancer using an alpha(v)beta(3-)integrin targeted agent. Gynecol Oncol 2013;128(3):590-5. 
43. Huang R, Vider J, Kovar JL, et al. Integrin alphavbeta3-targeted IRDye 800CW nearinfrared imaging of glioblastoma. Clin Cancer Res 2012;18(20):5731-40.

44. Keramidas M, Josserand V, Righini CA, Wenk C, Faure C, Coll JL. Intraoperative near-infrared image-guided surgery for peritoneal carcinomatosis in a preclinical experimental model. Br J Surg 2010;97(5):737-43.

45. Yokoyama J, Fujimaki M, Ohba S, et al. A feasibility study of NIR fluorescent imageguided surgery in head and neck cancer based on the assessment of optimum surgical time as revealed through dynamic imaging. Onco Targets Ther 2013;6:325-30.

46. Sandanaraj BS, Gremlich HU, Kneuer R, Dawson J, Wacha S. Fluorescent nanoprobes as a biomarker for increased vascular permeability: implications in diagnosis and treatment of cancer and inflammation. Bioconjug Chem 2010;21(1):93-101.

47. Maeda H. Tumor-selective delivery of macromolecular drugs via the EPR effect: background and future prospects. Bioconjug Chem 2010;21(5):797-802. 